\title{
Entanglement Preserving in Quantum Copying of Three-qubit Entangled State
}

\author{
TONG Zhao-Yang and KUANG Le-Man \\ Department of Physics, Hunan Normal University, Changsha 410081, China
}

\begin{abstract}
We study the degree to which quantum entanglement survives when a three-qubit entangled state is copied by using local and non-local processes, respectively, and investigate iterating quantum copying for the three-qubit system. There may exist inter-three-qubit entanglement and inter-two-qubit entanglement for the three-qubit system. We show that both local and non-local copying processes degrade quantum entanglement in the three-particle system due to a residual correlation between the copied output and the copying machine. We also show that the inter-two-qubit entanglement is preserved better than the inter-three-qubit entanglement in the local cloning process. We find that non-local cloning is much more efficient than the local copying for broadcasting entanglement, and output state via non-local cloning exhibits the fidelity better than local cloning.
\end{abstract}

PACS numbers: 03.75.Fi, 03.65.Bz, 32.80.Pj

Key words: entangled states, quantum entanglement, quantum copying

\section{INTRODUCTION}

The most fundamental difference between classical and quantum information is that while classical information can be copied perfectly, quantum information cannot. In particular, it follows from the no-cloning theorem [1] that one cannot create a perfect duplicate of an arbitrary qubit. Nevertheless, Buzek and Hillery and other authors [2, 3, 4, 5] have shown that imperfect copies can be made by a universal quantum cloning machine (UQCM), the outputs of which are identical. The price which must be paid is that there is a difference between the original input and the copies, because of residual entanglement between the machine and copies. However, not only similarity is lost during the cloning process. Perhaps even more import than the no-cloning feature of quantum mechanics is entanglement, first noted by Einstein-Podolsky-Rosen (EPR)and Schrödinger [6]. For decades, quantum entanglement has been the focus of much work in the foundations of quantum mechanics. In particular, it is associated with quantum nonseparability, the violation of Bell's inequalities, and the so-called EPR paradox. Beyond this fundamental aspect, creating and manipulating of entangled states are essential for quantum information applications. Among these applications are quantum teleportation [], quantum dense coding [8], quantum error correction [9], and quantum computational speedups [10], quantum cryptography [11], and quantum positioning and clock synchronization [12]. Hence, quantum entanglement has been viewed as an essential resource for quantum information processing. all of these applications depend upon the strength of quantum entanglement. If the cloning process applied to entangled subsystems is to be anything more than a basic curiosity, and is to find a practical application in the field of quantum information theory, it should be possible to obtain not only maximally accurate copies of the original state, but also copies which preserve entangling characteristic of the original state when the input state is an entangled state. Masiak and Knight [13] have shown that copies of entangled pair of qubits can be generated by using the UQCM.
In this paper, We will investigate the degree to which entanglement survives when a three qubit entangled state is copied by using local and non-local processes, respectively. We will show how these copying processes degrade quantum entanglement due to a residual correlation between the copied output and the copying machine. We will also show that entanglement is rapidly destroyed by the copying process.

\section{ENTANGLEMENT VARIATION AND STATE FIDELITY IN QUANTUM COPYING}

We consider quantum copying of a non-local state of a three-particle system consisting of a three qubits and assume that the three qubits are initially prepared in a entangled pure state expressed by

$$
|\psi\rangle=\cos \alpha|000\rangle+\sin \alpha|111\rangle .
$$

There are two approaches to realize quantum copying of the above entangled state. The first one is that each of the three original qubits is copied separately by using three different local copying machines. The second method is that the entangled state of the three qubits is treated as a state in a large Hilbert space and copied as a whole. The former is called as local cloning while the latter is regarded as non-local cloning.

We will measure quantum entanglement in a threeparticle system by making use of the entanglement tensor approach which is first proposed in Ref. [15] and further developed for a three-particle system by present authors [16]. Entanglement measures of a three-qubit system involve both an inter-three-qubit entanglement measure denoted by $E_{3}$ and an inter-two-qubit entanglement measure labelled by $E_{2}$. Based on entanglement tensors $M(1,2,3)$ and $M(m, n)(1 \leq m<n \leq 3)$ introduced in Ref. [15], entanglement measures $E_{3}$ and $E_{2}$ 
can be defined as follows

$$
\begin{aligned}
& E_{3}=\frac{1}{4} \sum_{i, j, k=1}^{3} M_{i j k}(1,2,3) M_{i j k}(1,2,3), \\
& E_{2}(m, n)=\frac{1}{3} \sum_{i, j=1}^{3} M_{i j}(m, n) M_{i j}(m, n),
\end{aligned}
$$

Here the entanglement tensors on the RHS of Eqs. (2) and (3) are defined by

$$
\begin{aligned}
M_{i j}(m, n)= & K_{i j}(m, n)-\lambda_{i}(m) \lambda_{j}(n) \\
M_{i j k}(1,2,3)= & K_{i j k}(1,2,3)-\lambda_{i}(1) M_{j k}(2,3) \\
& -\lambda_{j}(2) M_{i k}(1,3)-\lambda_{k}(3) M_{i j}(1,2) \\
& -\lambda_{i}(1) \lambda_{j}(2) \lambda_{k}(3),
\end{aligned}
$$

where $K_{i j k}(1,2,3), K_{i j}(m, n)$, and $\lambda_{i}(m)$ are the correlation tensors and coherence vectors defined in Ref. 16], respectively.

These measures are invariant under local unitary transformations of the subsystems and their values can change between 0 (unentangled states) and 1 (maximum entangled states). $E_{3}$ quantifies the three-qubit entanglement - The larger $E_{3}$ is, the stronger the three-qubit entanglement is. And $E_{2}(m, n)$ quantifies the entanglement between any two qubits $m, n$ in the three-qubit system. The larger $E_{2}(m, n)$ is, the stronger the entanglement between two qubit $m, n$ is. Making use of Eqs. (2-8) in Ref. 16] we can obtain the nonzero components of the coherence vector and correlation tensor

$$
\begin{aligned}
\lambda_{3}(1) & =\lambda_{3}(2)=\lambda_{3}(3)=-\cos (2 \alpha), \\
K_{33}(1,2) & =K_{33}(2,3)=K_{33}(1,3)=1, \\
K_{111} & =\sin (2 \alpha), \quad K_{333}=-\cos (2 \alpha), \\
K_{122} & =K_{212}=K_{221}=-\sin (2 \alpha) .
\end{aligned}
$$

From Eqs. (4) and (5) one can find the nonzero components of the entanglement tensor

$$
\begin{aligned}
M_{111} & =\sin (2 \alpha), \\
M_{333} & =2 \sin ^{2}(2 \alpha) \cos (2 \alpha), \\
M_{122} & =M_{212}=M_{221}=-\sin (2 \alpha), \\
M_{33}(1,2) & =M_{33}(2,3)=M_{33}(1,3) \\
& =\sin ^{2}(2 \alpha),
\end{aligned}
$$

while all other components of the entanglement tensor vanish. Then from Eqs. (2) and (3) we find the values of entanglement measures $E_{3}$ and $E_{2}(m, n)$ as follows

$$
\begin{aligned}
E_{3} & =\sin ^{2}(2 \alpha)\left(1+\sin ^{2} 2 \alpha \cos ^{2}(2 \alpha)\right), \\
E_{2}(1,2) & =E_{2}(2,3)=E_{2}(1,3)=\frac{1}{3} \sin ^{4}(2 \alpha),
\end{aligned}
$$

which indicate that the three-qubit GHZ state $|\psi\rangle=$ $(|000\rangle+|111\rangle) / \sqrt{2}$ is the maximally entangled threequbit state with the maximal value of the inter-threequbit entanglement $E_{3}=1$.
It is well known that there are two approaches to realize quantum copying of the entangled state given by Eq. (1). The first one is that each of the three original qubits is copied separately by using three different local copying machines. The second method is that the entangled state of the three qubits is treated as a state in a large Hilbert space and copied as a whole. The former is called as local cloning while the latter is regarded as non-local cloning. In what follows we will investigate entanglement variation and copying fidelity in the two types of quantum cloning processes.

\section{A. Local cloning}

A scheme which will achieve local cloning is described by the following process [14]: three distant parties share an entangled three qubit state $|\psi\rangle$. Each of them performs some local transformations on their own qubit using distant quantum copying machines. We assume that the three additional qubits, employed in the copying process, are initially unentangled. Three copiers separately make copies of the qubits by the following local unitary transformations [17]

$$
\begin{aligned}
U_{1}|0\rangle_{a_{0}}|0\rangle_{a_{1}}|X\rangle_{x}= & \sqrt{\frac{2}{3}}|00\rangle_{a_{0} a_{1}}|\uparrow\rangle_{x} \\
& +\sqrt{\frac{1}{3}}|+\rangle_{a_{0} a_{1}}|\downarrow\rangle_{x} \\
U_{1}|1\rangle_{a_{0}}|0\rangle_{a_{1}}|X\rangle_{x}= & \sqrt{\frac{2}{3}}|11\rangle_{a_{0} a_{1}}|\downarrow\rangle_{x} \\
& +\sqrt{\frac{1}{3}}|+\rangle_{a_{0} a_{1}}|\uparrow\rangle_{x}
\end{aligned}
$$

where $|+\rangle_{a_{0} a_{1}}=\left(|10\rangle_{a_{0} a_{1}}+|01\rangle_{a_{0} a_{1}}\right) / \sqrt{2}$. The system labelled by $a_{0}$ is the original (input) qubit, while the other system $a_{1}$ represents the target qubit onto which the information is copied. The states of the copying machine are labelled by $x$. The state space of the copying machine is two dimensional and we assume that it is always in the same state $|X\rangle_{x}$ initially. The result of the cloning process is an output state which is no longer a pure state, but is a mixed state described by the following density matrix

$$
\begin{aligned}
\hat{\rho}= & \frac{1+124 \cos ^{2} \alpha}{216}|000\rangle\langle 000| \\
& +\frac{1+124 \sin ^{2} \alpha}{216}|111\rangle\langle 111| \\
& +\frac{8 \sin \alpha \cos \alpha}{27}(|111\rangle\langle 000|+| 000\rangle\langle 111|) \\
& +\frac{5+20 \sin ^{2} \alpha}{216}(|110\rangle\langle 110|+| 011\rangle\langle 011| \\
& +|101\rangle\langle 101|)+\frac{5+20 \cos ^{2} \alpha}{216}(|100\rangle\langle 100| \\
& +|010\rangle\langle 010|+| 001\rangle\langle 001|) .
\end{aligned}
$$

We now calculate entanglement for the output state of local quantum copying described by above density oper- 
ator. Making use of Eqs. (2-8) in Ref. [16] we can obtain the nonzero coherence vectors and correlation tensors

$$
\begin{aligned}
\lambda_{3}(1) & =\lambda_{3}(2)=\lambda_{3}(3)=-\frac{2}{3} \cos (2 \alpha), \\
K_{33}(1,2) & =K_{33}(2,3)=K_{33}(1,3)=\frac{4}{9} \\
K_{111} & =\frac{8}{27} \sin (2 \alpha), \quad K_{333}=\frac{8}{27} \cos (2 \alpha), \\
K_{122} & =K_{212}=K_{221}=-\frac{8}{27} \sin (2 \alpha) .
\end{aligned}
$$

From Eqs. (4) and (5) one can find the following entanglement tensors

$$
\begin{aligned}
M_{111} & =\frac{8}{27} \sin (2 \alpha), \\
M_{333} & =\frac{16}{27} \sin ^{2}(2 \alpha) \cos (2 \alpha), \\
M_{122} & =M_{212}=M_{221}=-\frac{8}{27} \sin (2 \alpha), \\
M_{33}(1,2) & =M_{33}(2,3)=M_{33}(1,3) \\
& =\frac{4}{9} \sin ^{2}(2 \alpha),
\end{aligned}
$$

while all other components of the entanglement tensor vanish. Then from Eqs. (2) and (3) we find the values of $E_{3}$ and $E_{2}(m, n)$ entanglements as follows

$$
\begin{aligned}
E_{3} & =\frac{64}{729} \sin ^{2}(2 \alpha)\left(1+\sin ^{2} 2 \alpha \cos ^{2}(2 \alpha)\right), \\
E_{2}(1,2) & =E_{2}(2,3)=E_{2}(1,3)=\frac{16}{243} \sin ^{4}(2 \alpha),
\end{aligned}
$$

which indicate that both the inter-three-qubit entanglement and inter-two-qubit entanglement in a three qubit system characterized by $E_{3}$ and $E_{2}(m, n)$, respectively, can be broadcasted via local quantum cloning, since the amount of $E_{3}$ and $E_{2}(m, n)$ entanglements are nonzero, i.e., $E_{3} \neq 0, E_{2}(m, n) \neq 0$ except $\alpha=0$ or $\alpha=\frac{\pi}{2}$. In particular, when $\alpha=\pi / 4$, we have $E_{3}=64 / 729$ and $E_{2}(n, m)=16 / 243$ which recover the result in Ref. 16] for quantum copying of the GHZ state.

Comparing remaining $E_{3}$ and $E_{2}(m, n)$ entanglements after local cloning given by Eqs. (38) and (39) with the original $E_{3}$ and $E_{2}(m, n)$ entanglements before local cloning given by Eqs. (14) and (15), we can find that both $E_{3}$ and $E_{2}(m, n)$ entanglements are reduced in the local cloning process. The remaining $E_{3}$ entanglement after local cloning is only 8.76 percent of the original $E_{3}$ entanglement, and the remaining $E_{2}$ entanglement after local cloning is 19.8 percent of the original $E_{2}$ entanglement. Therefore, the $E_{2}$ entanglement is preserved better than the $E_{3}$ entanglement in the local cloning process.

\section{B. Non-local cloning}

The entangled state given in Eq. (3) $|\psi\rangle$ can also be cloned non-locally [18]. In this case the entangled state of the three-qubits is treated as a state in a larger Hilbert
E3

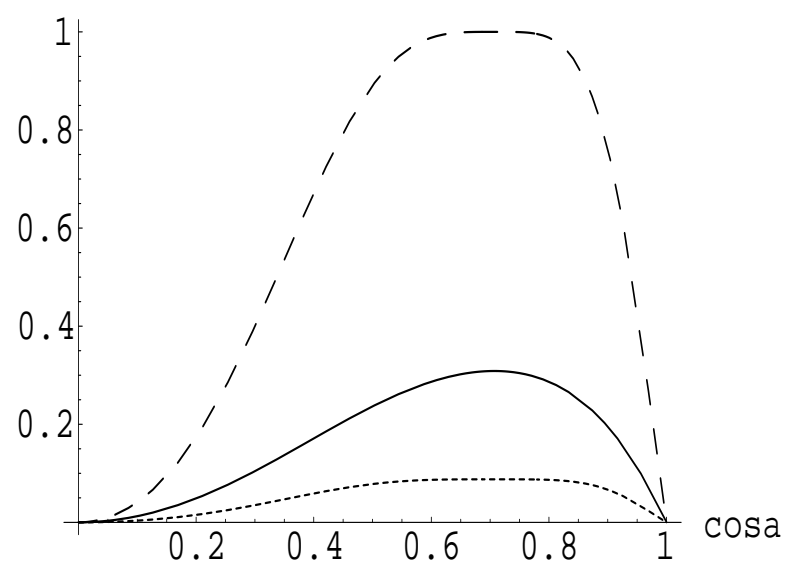

FIG. 1: $E_{3}$ entanglement of the three-qubit pure state $|\psi\rangle$ (dashed curve) and remaining $E_{3}$ entanglement after the first step of local (dotted curve) and non-local (solid curve) cloning.

space and cloned as a whole. The non-local quantum copying machine [19] is an $N$ dimensional quantum system, and we shall let $\left|X_{i}\right\rangle_{x}(i=1, \cdots, N)$ be a set of orthonormal basis of the copying machine Hilbert space. This copier is initially prepared in a particular state $|X\rangle_{x}$. The action of the cloning transformation can be specified by a unitary transformation acting on the basis vectors of the tensor product space of the original quantum system $\left|\phi_{i}\right\rangle_{a_{0}}$, the copier, and an additional $N$ dimensional system which is to become the copy (which is initially prepared in an arbitrary state $|0\rangle_{a_{1}}$ ). The corresponding transformation $U_{2}$ is given by

$$
\begin{aligned}
U_{2}\left|\phi_{i}\right\rangle_{a_{0}}|0\rangle_{a_{1}}|X\rangle_{x}= & c\left|\phi_{i}\right\rangle_{a_{0}}\left|\phi_{i}\right\rangle_{a_{1}}\left|X_{i}\right\rangle_{x} \\
& +d \sum_{j \neq i}^{N}\left(\left|\phi_{i}\right\rangle_{a_{0}}\left|\phi_{j}\right\rangle_{a_{1}}\right. \\
& \left.+\left|\phi_{j}\right\rangle_{a_{0}}\left|\phi_{i}\right\rangle_{a_{1}}\right)\left|X_{j}\right\rangle_{x}(29)
\end{aligned}
$$

where $i=1, \cdots, N, c^{2}=2 /(N+1)$, and $d^{2}=1 / 2(N+1)$.

The final state of the three-qubit copies at the output of the cloning machine is given by the following density operator

$$
\begin{aligned}
\hat{\rho}= & \frac{1+10 \cos ^{2} \alpha}{18}|000\rangle\langle 000| \\
& +\frac{1+10 \sin ^{2} \alpha}{18}|111\rangle\langle 111| \\
& +\frac{5 \sin \alpha \cos \alpha}{9}(|111\rangle\langle 000|+| 000\rangle\langle 111|) \\
& +\frac{1}{18}(|110\rangle\langle 110|+| 011\rangle\langle 011|+| 101\rangle\langle 101| \\
& +|100\rangle\langle 100|+| 010\rangle\langle 010|+| 001\rangle\langle 001|) .(30
\end{aligned}
$$

Now we check whether entanglement is broadcasted. 
Through lengthy calculations, we obtain the nonzero components of the coherent vector and correlation tensor

$$
\begin{aligned}
\lambda_{3}(1) & =\lambda_{3}(2)=\lambda_{3}(3)=-\frac{5}{9} \cos (2 \alpha), \\
K_{33}(1,2) & =K_{33}(2,3)=K_{33}(1,3)=\frac{5}{9}, \\
K_{111} & =\frac{5}{9} \sin (2 \alpha), \quad K_{333}=-\frac{5}{9} \cos (2 \alpha), \\
K_{122} & =K_{212}=K_{221}=-\frac{5}{9} \sin (2 \alpha),
\end{aligned}
$$

which leads to the following non-vanishing components of entanglement tensor

$$
\begin{aligned}
M_{111} & =\frac{5}{9} \sin (2 \alpha) \\
M_{333} & =\frac{10}{27}\left(1-\frac{25}{27} \cos ^{2}(2 \alpha)\right) \cos (2 \alpha), \\
M_{122} & =M_{212}=M_{221}=-\frac{5}{9} \sin (2 \alpha) \\
M_{33}(1,2) & =M_{33}(2,3)=M_{33}(1,3) \\
& =\frac{5}{9}-\frac{25}{81} \cos ^{2}(2 \alpha) .
\end{aligned}
$$

Substituting Eqs.(35138) into Eqs. (2) and (3), we find the values $E_{3}$ and $E_{2}(m, n)$ to be

$$
\begin{aligned}
E_{3}= & \frac{25}{81} \sin ^{2}(2 \alpha) \\
& +\frac{25}{729}\left(1-\frac{25}{27} \cos ^{2}(2 \alpha)\right)^{2} \cos ^{2} 2 \alpha \\
E_{2}(1,2)= & E_{2}(2,3)=E_{2}(1,3) \\
= & \frac{25}{243}\left(1-\frac{5}{9} \cos ^{2}(2 \alpha)\right)^{2} .
\end{aligned}
$$

From which we see that both $E_{3}$ and $E_{2}$ entanglements can be broadcasted via the non-local process. Obviously, when $\alpha=\pi / 4$, we have $E_{3}=25 / 81$ and $E_{2}(n, m)=$ 25/243 which recover the result in Ref. [16] for quantum copying of the GHZ state.

We now numerically discuss entanglement variation in the local and non-local copying processes with respect to the values of $\cos \alpha$. In Fig. 1 and Fig. 2 we plot the $E_{3}$ entanglement and $E_{2}$ entanglement in the three-qubit input state $|\psi\rangle$ and the three-qubit output states obtained as the result of local and non-local copying of the state $|\psi\rangle$, respectively. From Fig.1 and Fig.2 we can see that both local and non-local copy can broadcast entanglement in the three-qubit system. And we find that non-local cloning is much more efficient than the local copying process for broadcasting entanglement. In the local cloning case, the amount of both $E_{3}$ entanglement and $E_{2}$ entanglement of the copied state is less than that of the original state for all values of $\cos \alpha$. In the non-local cloning case, the amount of $E_{3}$ entanglement of the copied state is always less than that of the original state for all values of $\cos \alpha$ while the

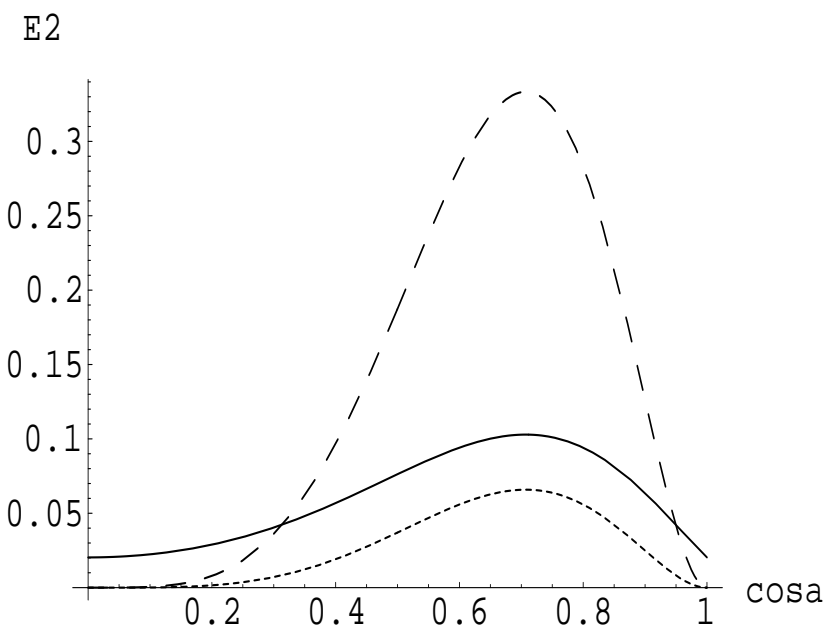

FIG. 2: $E_{2}$ entanglement of the three-qubit pure state $|\psi\rangle$ (dashed curve) and remaining $E_{2}$ entanglement after the first step of local (dotted curve) and non-local (solid curve) cloning.

amount of $E_{2}$ entanglement of the copied state is less than that of the original state for most range of $\cos \alpha$, i.e., $0.33065<\cos \alpha<0.95287$. In other words, the remaining $E_{2}$ entanglement after the non-local cloning is amplified versus the initial state when $\cos \alpha<0.33065$ or $\cos \alpha>0.95287$. This inter-two-particle entanglement amplification in a three-particle system is originated from inter-two-particle, and particle-copier interaction. However, inter-three-qubit entanglement can not be amplified for both local and nonlocal cloning processes. Hence this does not violate the well known fact that copying a quantum system does not increase the information obtainable about the originals.

Finally, It is interesting to compare the fidelity $F_{1}$ of the output density operator after local copying relative to $|\psi\rangle$ with the fidelity $F_{2}$ of the output density operator after non-local copying relative to $|\psi\rangle$. The fidelity of a density matrix $\rho$ relative to $|\psi\rangle$ is defined by $F=\langle\psi|$ $\rho|\psi\rangle$. From Eqs. (10), (61), and (30) we get that

$$
\begin{aligned}
& F_{1}=\frac{125}{216}-\frac{15}{27} \sin ^{2} \alpha \cos ^{2} \alpha, \\
& F_{2}=\frac{11}{18} .
\end{aligned}
$$

From which we find that the fidelity $F_{2}$ of the output state after non-local copying is independent of the values of $\cos \alpha$. This means that whether the initial state is like, the fidelity of the output is definite. However, in the local copying process, the fidelity $F_{1}$ depends upon the initial state. From Eq. (411), we see that when $\cos \alpha=0$ or 1 , the output state is the closest to the original state. When $\cos \alpha=\sqrt{2} / 2$, the output state is the farthest to the original state. In Fig.3 we plot the fidelity with respect to $\cos \alpha$ for local and nonlocal cloning cases, respectively. 


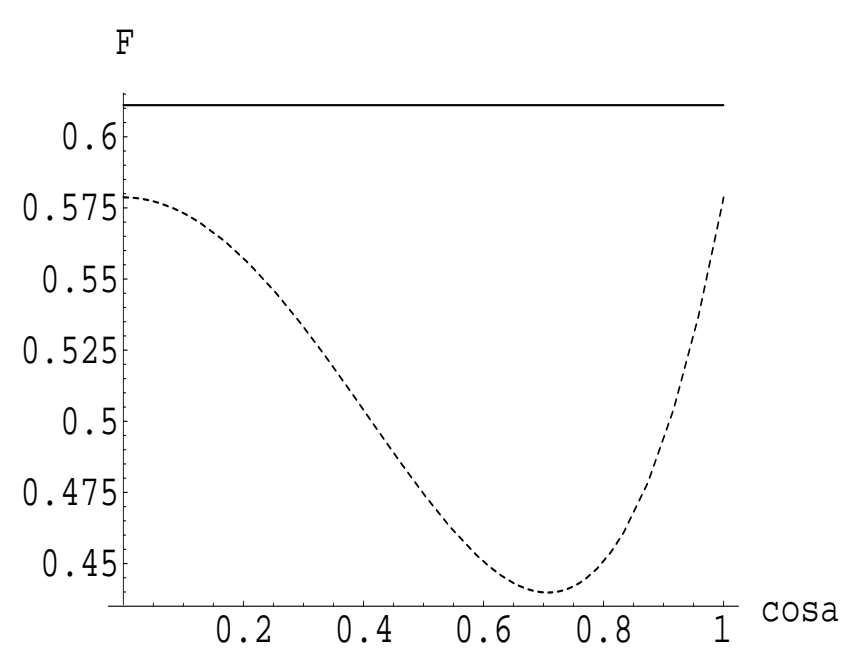

FIG. 3: Fidelity of the output state after local cloning (dotted curve) and non-local cloning (solid curve).

From Fig. 3 it can be seen that the fidelity in the nonlocal cloning process is always greater than that in the local cloning process, i.e., $F_{2}>F_{1}$. This means that the output state after non-local copying is closer to the original state than the output state after local copying.

\section{REPETITION OF NON-LOCAL CLONING}

We can see from the previous section that both local and non-local copying are not rather efficient process from the point of view of preserving entanglement. Even in the case when the maximally inseparable state, i.e. GHZ state where $\alpha=\pi / 4$, is the input state of the cloning process, only a small amount of entanglement survives the cloning. It is an interesting question to ask what will happen when the output state of the copier is used as an input state in the next step of a sequence of cloning process. In particular, we are interested in discovering how fast the entanglement decreases when the copying is iterated. We restrict ourself in the case of nonlocal cloning, because this scheme is much more effective as we saw above, and results obtained in this case can be treated as an upper bound for all other schemes.

The final state of the copying process is a mixed state, described by the density matrix, Eq. (301). This density matrix cannot be used directly as input data in computations, because the non-local copying scheme works straightforwardly only when an input state is initially in a pure, potentially entangled state. The density matrix should be first converted to a form which allows us to perform the second cloning. It turns out that a simple diagonalization of the density matrix is sufficient. In this new diagonal basis, the density matrix is given by the mixture of projection operators

$$
\hat{\rho}=\sum_{j=1}^{8} \alpha_{j}\left|\varphi_{j}\right\rangle\left\langle\varphi_{j}\right| .
$$

The weights $\alpha_{j}$ in the decomposition are the eigenvalues of the density matrix $\hat{\rho}$ and the vectors $\left|\varphi_{j}\right\rangle$ are the normalized eigenvectors of $\hat{\rho}$. Each vector $\left|\varphi_{j}\right\rangle$ can be cloned separately. The mixture of the resultant density matrices taken with the weights $\alpha_{j}$ is the result of the second cloning process.

As a specific example of iterating copying, in what follows we consider repetition of non-local quantum cloning for the GHZ state given by Eq. (II) through taking $\alpha=\pi / 4$. After the first non-local cloning, from Eq. (30) we express the density operator of the out state of the original three input qubits as the form of the state 43 with the coefficients

$$
\alpha_{8}=\frac{11}{18}, \quad \alpha_{i}=\frac{1}{18}(i=1,2, \cdots, 7),
$$

and eigenvectors of the out-state density operator given by

$$
\begin{aligned}
\left|\varphi_{1}\right\rangle & =\frac{1}{\sqrt{2}}(|000\rangle-|111\rangle), \quad\left|\varphi_{2}\right\rangle=|110\rangle \\
\left|\varphi_{3}\right\rangle & =|101\rangle, \quad\left|\varphi_{4}\right\rangle=|100\rangle \\
\left|\varphi_{5}\right\rangle & =|011\rangle, \quad\left|\varphi_{6}\right\rangle=|010\rangle \\
\left|\varphi_{8}\right\rangle & =\frac{1}{\sqrt{2}}(|000\rangle+|111\rangle), \quad\left|\varphi_{7}\right\rangle=|001\rangle
\end{aligned}
$$

TABLE I: Entanglement $E_{3}$ and $E_{2}$ of clones of the GHZ state as a function of the number of cloning steps.

The state described by the density operator (43) is the input state of the second copying. We now separately copy the eigenstates of the density operator (43), i.e., $\left.\varphi_{i}\right\rangle$, via non-local process. After the second copying, we arrive at the output state expressed by the following density operator

$$
\begin{aligned}
\hat{\rho}= & \frac{54}{13}(|000\rangle\langle 000|+| 111\rangle\langle 111|) \\
& +\frac{25}{162}(|111\rangle\langle 000|+| 000\rangle\langle 111|) \\
& +\frac{7}{81}(|110\rangle\langle 110|+| 011\rangle\langle 011|+| 101\rangle\langle 101| \\
& +|100\rangle\langle 100|+| 010\rangle\langle 010|+| 001\rangle\langle 001|),(49)
\end{aligned}
$$

which leads to the following entanglement tensors

$$
\begin{aligned}
M_{122} & =-M_{111}=M_{212}=M_{221}=-\frac{25}{81}, \\
M_{33}(1,2) & =M_{33}(2,3)=M_{33}(1,3)=\frac{25}{81} .
\end{aligned}
$$


Hence after finishing the second copying, entanglement of the three-qubit system becomes

$$
\begin{aligned}
E_{3} & =\left(\frac{25}{81}\right)^{2}, \\
E_{2}(1,2) & =E_{2}(2,3)=E_{2}(1,3)=\frac{1}{3}\left(\frac{25}{81}\right)^{2} .
\end{aligned}
$$

From above calculations we can see that entanglement in the three-qubit system is further reduced after the second copying. Repeating the above procedure we can investigate changes in entanglement $E_{3}$ and $E_{2}$ at each stage of iterating copy process. In Table 1 we show the entanglement measures $E_{3}$ and $E_{2}(m, n)$ of the clones of the maximally entangled GHZ state for the case of the first five stages of the cloning. From the this table, we see that the entanglement decreases extremely rapidly, and after just six iterations the copy of the GHZ state, the resultant entanglement goes to zero.

\section{CONCLUSIONS}

In conclusion, we have investigated remaining entanglement in quantum copying and iterating quantum copying of a three-qubit system by using the entanglement tensor approach. Specifically, we have studied the remaining inter-two-qubit and inter-three-qubit entanglements in the local and non-local cloning processes, respectively. we have shown that entanglement of the three-qubit pure state can be locally or non-locally copied with the help of local quantum copiers or non-local quantum copiers, respectively, and that the amount of entanglements of the resultant copiers is reduced. It has been found that it is more efficient to preserve the entanglement by using non-local methods. The amount of entanglement decreases rapidly with the increase of the number of copying times in the iterating quantum copying. The amount of both $E_{3}$ and $E_{2}$ entanglements approach zero after six-times iterating quantum copying. It means that even qubits, which are copies of copies of copies of copies of copies of copies of the GHZ state are already in local state. They do not have any nonclassical correlations and are useless as a resource in cryptographic conferencing or in multi-partite generalizations of super-dense coding [20]. This reduction is due to a residual entanglement between the copies and the quantum copying machine. We have also shown that the inter-two-qubit entanglement is preserved better than the inter-three-qubit entanglement in the local cloning process while non-local cloning is much more efficient than the local cloning for broadcasting entanglement, and output state via non-local cloning exhibits the fidelity better than local cloning.

\section{ACKNOWLEDGMENTS}

This work was supported in part by the National Natural Science Foundation, EYTF of the Educational Department of China, and Hunan Province STF.
[1] W. K. Wootters and W. H. Zurek, Nature 299 (1982) 802.

[2] D. Mozyrsky, V. Privman, and M. Hillery, Phys. Lett. A 226 (1997) 253.

[3] V. Buzek and M. Hillery, Phys. Rev. A 54 (1996) 1844.

[4] N. Gisin and S. Massar, Phys. Rev. Lett. 79 (1997) 2153.

[5] D. Bruss, A. Ekert, and C.Macchiavello, Phys. Rev. Lett. 81 (1998)2598.

[6] A. Einstein, B. Podolsky and N. Rosen, Phys. Rev. 47 (1935) 777; E. Schrödinger, Naturwissenschaften, 23 (1935) 807; 823; 844.

[7] C. H. Bennett, G. Brassard, C. Crépeau, R. Jozsa, A. Peres, and W. K. Wootters, Phys. Rev. Lett. 70 (1993) 1895; D. Bouwmeester, J.-W. Pan, K. Mattle, M.Eibl, H. Weinfurter, A. Zeilinger, Nature 390 (1997) 575.

[8] C. H. Bennett and S. J. Wiesner, Phys. Rev. Lett. 69 (1992) 2881.

[9] P. W. Shor, Phys. Rev. A 52 (1995) 2493.

[10] D. Deutsch, Proc. R. Soc. London, A 425 (1989) 73.
[11] T. Jennewein, C. Simon, G. Weihs, H. Weinfurter, and A. Zeilinger, Phys. Rev. Lett. 84 (2000) 4729.

[12] V. Giovannetti, S. LIoyd, and L. Maccone, Nature 412 (2001)417; V. Giovannetti, S. Lloyd, and L. Maccone, Phys. Rev. A 65 (2002) 022309.

[13] P. Masiak and P. L. Klight, quant-ph/9808043

[14] V. Buzek, V. Vedral, M. B. Plenio, P. L. Knight, and M. Hillery, Phys. Rev. A 55 (1997) 3327.

[15] J. Schlienz and G. Mahler, Phys. Rev. A 52 (1995) 4396.

[16] Z.-Y. Tong and L.-M. Kuang, Chin. Phys. Lett. 17 (2000) 469.

[17] V. Buzek and M. Hillery, Phys.Rev. A 54 (1996) 1844; D. Bruß, D.P. DiVincenzo, A. Ekert, C.A. Fuchs, C. Macchiavello, and J.A. Smolin, Phys. Rev. A 57 (1998) 2368.

[18] V. Buzek and M. Hillery, quant-ph/9801009

[19] R. F. Werner, Phys. Rev. A58 (1998) 1827.

[20] S. Bose, V. Vedral and P. L. Klight, Phys. Rev. A. 57 (1998) 822. 